\title{
Rapid Progression of Neonatal Respiratory Distress from Laryngeal Cleft Type I: A Case Report
}

\author{
Yumi Doi, $M D, P h D^{1,2^{*}}$ and Eiji Nishijima, $M D^{3}$ \\ ${ }^{1}$ Department of Anesthesia, Takatsuki General Hospital, Takatsuki, Japan \\ ${ }^{2}$ Pediatric Intensive Care Unit, Takatsuki General Hospital, Takatsuki, Japan \\ ${ }^{3}$ Department of Pediatric Surgery, Takatsuki General Hospital, Takatsuki, Japan
}

*Corresponding author: Yumi Doi, Department of Anesthesia, Pediatric Intensive Care Unit, Takatsuki General Hospital, 1-3-13 Kosobe-cho, Takatsuki, Osaka 5691192, Japan, Tel: +81-72-681-3801, Fax: +81-72-682-3834

\begin{abstract}
A one-month-old boy showed cyanosis and severe retraction on crying. He underwent diagnostic rigid bronchoscopy under general anesthesia, maintaining spontaneous ventilation. Rigid bronchoscopy revealed laryngeal cleft type I and excessive mucosa in the interarytenoid space that protruded into the subglottic area, very unusual findings in laryngeal cleft. His clinical condition quickly deteriorated and tracheotomy was performed to prevent complete airway obstruction in the few days until surgical repair of the cleft. Tracheotomy is unnecessary in most cases of the laryngeal cleft type I, but it may be needed if the patient's airway is severely compromised.
\end{abstract}

\section{Keywords}

Laryngeal cleft type I, Neonate, Subglottic, Rigid bronchoscopy, Tracheotomy

\section{Introduction}

Laryngeal cleft (LC) is a rare congenital malformation with an incidence of 1 in 10,000-20,000 live births [1]. Intensity of clinical symptoms typically correlates with the type of cleft. LC has been classified according to the distal end of the cleft, which extends to the level of the true vocal cords (type 1), into the cricoid cartilage (type $2)$, through the cricoid cartilage and into the cervical trachea (type 3), and into the thoracic trachea (type 4). LC types 0 and I may be asymptomatic or show only mild symptoms, so incidence is possibly underestimated [2,3]. Moreover, LC frequently presents clinical symptoms that can be misattributed to more common diseases, so it is often undiagnosed [4]. Pediatric patients with low- grade LC show stridor, toneless or hoarse crying and swallowing disorders. Aspiration, coughing, dyspnea, and cyanosis during feeding and crying are possible, while severe respiratory symptoms are rare. LC types 0 and I are usually treated medically, and only those with failed medical treatment tend to require surgery. We describe a neonate with LC type I with severe respiratory distress early in life that required immediate tracheotomy as airway management. Written consent has been obtained from the patient's parent.

\section{Case Description}

A one-month-old boy weighing 4088g had hoarse crying that induced cyanosis on crying. He was born at 39 weeks and 5 days of gestation, with a birthweight of $3330 \mathrm{~g}$. Although faint wheezing was noted from birth, he was discharged because he showed no signs of swallowing disorders, coughing or dyspnea, and bodyweight gain was considered to be within normal limits. Respiratory retraction and wheezing gradually worsened, and cyanosis was noticed during feeding and crying. He was seen at a local hospital at 4 weeks of age. Percutaneous oxygen saturation $\left(\mathrm{SpO}_{2}\right)$ was $60 \%$ while crying, so he was transferred to our hospital for diagnosis and treatment. Chest auscultation was normal and $\mathrm{SpO}_{2}$ was $100 \%$ without ventilatory support on rest. When he cried, however, there was severe retraction and chest deformity, and $\mathrm{SpO}_{2}$ decreased to $60 \%$. The patient was transferred to the pediatric intensive care unit (PICU) for close observation and prompt treatment in case of further respiratory deterioration. He was 


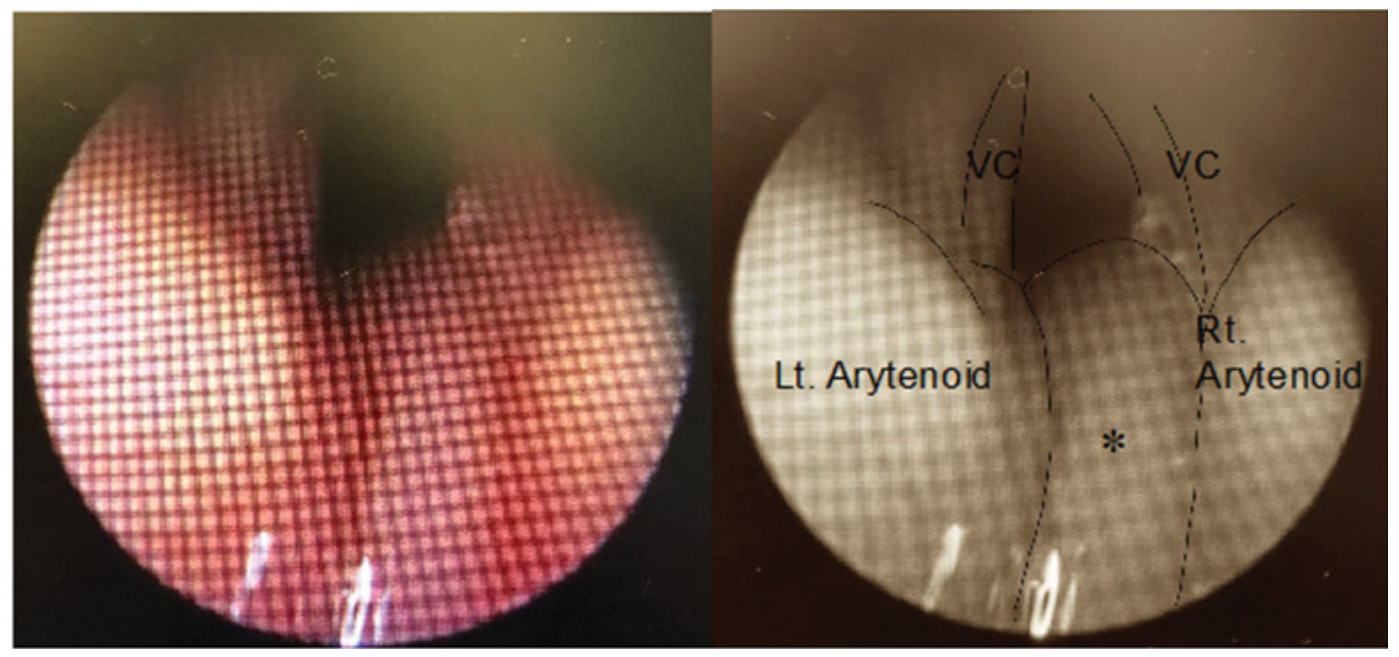

Figure 1: Endoscopic view of a laryngeal cleft.

Rigid bronchoscopy was performed under general anesthesia, maintaining spontaneous respiration. Laryngeal cleft and excessive interarytenoid mucosa were observed. Under suppressed spontaneous breathing, the airway seemed to be secure. Without anesthesia the mucosa may have prolapsed further, causing cyanosis.

*: interarytenoid mucosa; VC: Vocal Cord.

fed $80-100 \mathrm{ml}$ of milk every three hours orally with respiratory effort, but it was not aspirated. Chest X-ray showed that the lung fields were clear, with no signs of aspiration pneumonia, and fiberoptic laryngoscopy detected no major anomalies. Echocardiography showed a small muscular ventricular septum defect. Computed tomography showed apparently physiological low grade stenosis in the subglottic area. There was no laryngomalacia, laryngeal palsy, hemangioma, glossal cyst, structural abnormality of vessels such as pulmonary artery sling, or other congenital cardiac abnormalities. After preoperative evaluation and according to the clinical course in PICU, differential diagnoses included congenital subglottic stenosis and very small subglottic hemangioma/tumor undetectable by enhanced CT scan. For further examination and treatment, rigid bronchoscopy was scheduled under general anesthesia maintaining spontaneous respiration, to be followed by tracheotomy under tracheal intubation if necessary. Anesthesia comprised continuous administration of propofol and ketamine and topical anesthetic use of lidocaine, maintaining spontaneous ventilation. Laryngomalacia and laryngeal palsy were not observed. Unexpectedly, the interspace between bilateral arytenoids was wide open, and excessive mucosa from the posterior wall had prolapsed into the subglottic area and was obstructing the airway (Figure 1). We diagnosed LC, but were uncertain whether it was type I or II. Assessment of the entire respiratory tract showed no anomalies in the lower respiratory tract. We expected that patient growth would ease respiratory symptoms and decided there was no need for surgical treatment. The patient returned to PICU after emergence from anesthesia without any airway devices in situ. After assessment by rigid bronchoscopy, there was increase of oral secretion and the patient required

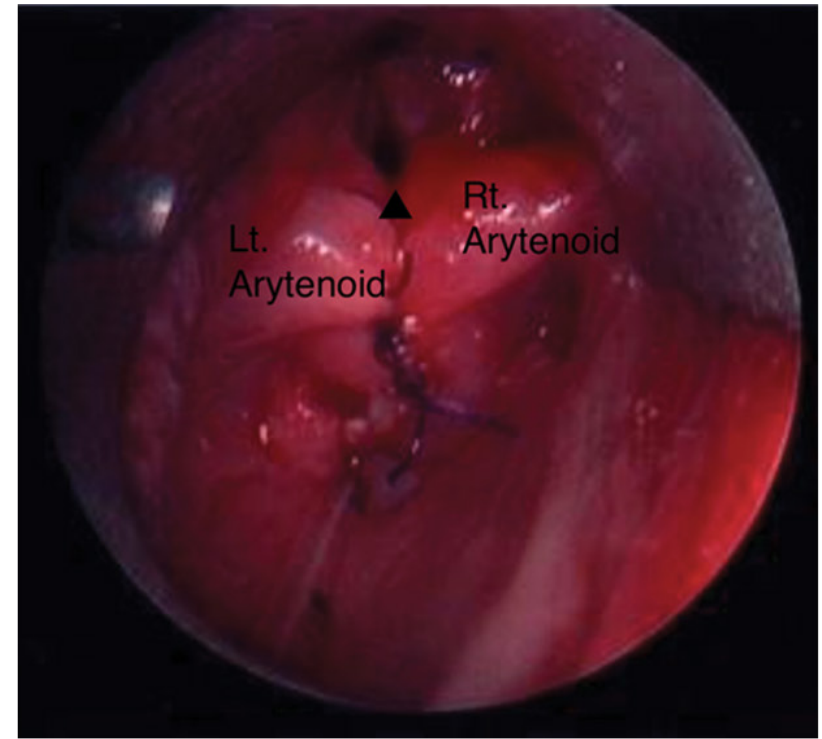

Figure 2: Endoscopic surgical repair of a laryngeal cleft. After excessive mucosa was evaporated by YAG laser, bilateral arytenoids were sutured with two stiches under suspension laryngoscopy.

$\Delta$ : airway

increased respiratory effort. $\mathrm{SpO}_{2}$ decreased to $50 \%$ when crying, which did not return to baseline without supplemental oxygen or positive airway pressure assistance. There was risk of complete airway tract blockage because neonatal airway tracts are especially narrow, and because mucosal protrusion critically impacts airway patency. A few days after assessment by first rigid bronchoscopy, tracheotomy and second rigid bronchoscope assessment were scheduled. Anesthesia was inducted by midazolam and fentanyl, followed by rocuronium bromide after confirmation of easy manual ventilation. His trachea was intubated easily with a cuffed 
$3.5 \mathrm{~mm}$ tracheal tube. After tracheotomy, second rigid bronchoscopy was performed, using suspension. Final diagnosis was LC type I. One month later, endoscopic repair of LC 1 was performed (Figure 2).

\section{Discussion}

In this unusual case, the neonatal patient showed no apparent clinical symptoms and seemingly required no treatment until one month of age. His respiratory condition then quickly deteriorated, however, and he suddenly required urgent surgical tracheotomy.

LC type I can be managed medically or surgically, depending on the degree of severity and morbidity. In symptomatic cases, early presentation can include feeding problems, choking, chronic coughing, wheezing, cyanotic spells and apnea. While surgery should be considered only for those whose medical treatment failed, the recommended duration and specifics of conservative management have varied greatly in the literature [2,5]. Decisions of how, when, and how long to initiate conservative therapy often depend on the individual clinician. Factors supporting surgical repair of LC type I are clinically-apparent aspiration with feeding, severity of pulmonary status, imaging studies and significant comorbid conditions [5]. Our patient did not show apparent aspiration, never experienced pneumonia, and had no comorbidities. Cyanotic spells and apparent life-threatening episodes were observed, however, and their severity and frequency increased dramatically after rigid bronchoscopy. Tracheotomy is rarely necessary [6], but in our case of a neonate with LC type I that previously did not need medical treatment, surgical intervention without attempting conservative management was necessary because of life-threating symptoms.

Reported ages at the diagnosis of LC type I vary widely. Most studies report a median age of diagnosis at between 12 and 24 months of age [2]. Parsons, et al. reported the average age at onset of symptoms as 4.6 months and diagnosis of LC type I was 3 years and 3 months [3]. This discrepancy is thought to imply variation in diagnostic techniques and in the recognition of the disease [7]. Diagnosis of LC type I is made based on various diagnostic methodologies, such as chest $X$-ray, modified barium swallow, flexible fiberoptic laryngoscopy and functional endoscopic evaluation of swallowing, echocardiography, chest CT scan and magnetic resonance imaging. Endoscopic assessment is essential for diagnosis of LC. When the current patient was referred to our hospital, the cause of cyanosis and stridor was still unknown even after chest X-ray, chest plain and enhanced CT scans and echocardiography. We performed flexible laryngoscopy, but the cause of symptoms was still unclear. The next day, the patient underwent rigid bronchoscopy under general anesthesia maintaining spontaneous breathing for further examination. His respiratory status worsened after this. One possible mechanism of respiratory deterioration is aspiration during rigid bronchoscopy resulting in microatelectasis $[8,9]$. General anesthesia is necessary for rigid bronchoscopy, but to some extent cough reflex is suppressed by anesthetics, and there may be aspiration of small amounts of oral secretions. Aspiration will impair oxygenation, trigger coughing and increase respiratory effort. Especially in neonates, respiratory muscles are vulnerable and muscle fatigue can easily lead to respiratory deterioration. The current gold standard treatment is endoscopic repair of the posterior laryngeal cleft under general anesthesia that maintains spontaneous respiration ("tubeless anesthesia") using suspension laryngoscope. However, our surgical team selected tracheotomy under general anesthesia before intervention of the cleft in consideration of the patient's condition after rigid bronchoscopy.

Compared with previous reports [4,5], our patient was diagnosed and needed surgical treatment at a relatively early stage of life. Gastroesophageal reflux regurgitation disease (GERD) is physiologically observed in neonates; about $70-85 \%$ have GERD within the first two months of life [10]. GERD leads to edematous changes of the larynx [11]. Excess mucosa herniating into the cleft is suggested to provide some protection against aspiration [12]. On the other hand, this mucosal herniation has also been suggested as a cause of stridor and airway obstruction. In our patient, the presence of the excess mucosa in the interarytenoid space may have caused the mucosa to be in contact with gastric acid for a longer period, resulting in more marked swelling. Airway narrowing by mucosal swelling of the glottis caused negative pressure of the airway on respiration, according to Bernoulli's theorem. The soft structure of the glottis was invaginated into the trachea due to strong negative pressure. The respiratory muscles were weak and negative pressure was insufficiently generated at birth. As the child grew, the respiratory muscles gradually started to generate a stronger negative pressure, which exacerbated the symptoms. This patient's LC type I was congenital, but he showed no clinical symptoms before discharge with the exception of very faint wheezing at birth. The gradual worsening of the symptoms can be attributed to two mechanisms: Interarytenoid mucosa swelling by exposure to gastric acid and negative airway pressure by respiratory muscles.

\section{Conclusion}

Respiratory deterioration in our patient was thought to be caused by involvement of gastric acid and increasingly negative intrathoracic pressure due to mucosal swelling and the development of respiratory muscles after birth.

Rigid bronchoscopy is necessary for accurate diagnosis of LC and its severity. Surgical treatment to secure the airway may be necessary if a neonate has respiratory failure, even if the $L C$ is type I and symptoms are expected to reduce as the child grows. 


\section{Acknowledgements}

We acknowledge proofreading and editing by Benjamin Phillis at the Clinical Study Support Center, Wakayama Medical University.

\section{Conflict of Interest}

The authors declare no conflict of interest.

\section{Financial Support}

None.

\section{Informed Consent}

Written informed consent was obtained from the patient's parents for publication.

\section{References}

1. Watters K, Ferrari L, Rahbar R (2013) Minimally invasive approach to laryngeal cleft. Laryngoscope 123: 264-268.

2. Isaac A, El-Hakim H (2019) Type 1 laryngeal cleft and feeding and swallowing difficulties in infants and toddlers: A Review. Clin Otolaryngol 44: 107-113.

3. Watters K, Russell J (2003) Diagnosis and management of type 1 laryngeal cleft. Int J Pediatr Otorhinolaryngol 67: 591-596.

4. Parsons DS, Stivers FE, Giovanetto DR, Phillips SE (1998) Type I posterior laryngeal clefts. Laryngoscope 108: 403410.
5. Rahbar R, Chen JL, Rosen RL, Lowry KC, Simon DM, et al. (2009) Endoscopic repair of laryngeal cleft type I and type II: when and why? Laryngoscope 119: 1797-1802.

6. Johnston DR, Watters K, Ferrari LR, Rahbar R (2014) Laryngeal cleft: Evaluation and management. Int J Pediatr Otorhinolaryngol 78: 905-911.

7. Rutter MJ, Azizkhan RG (2014) Chapter 21 Posterior Laryngeal Cleft. In: Ziegler MM, Azizkhan RG, von Allmen D, Weber TR, Operative Pediatric Surgery. (2 $2^{\text {nd }}$ edn), McGrawHill Education, New York, NY: 307-316.

8. Londino AV, Jagannathan N (2019) Anesthesia in diagnostic and therapeutic pediatric bronchoscopy. Otolaryngol Clin North Am 52: 1037-1048.

9. Ferrari LR, Zurakowski D, Solari J, Rahbar R (2013) Laryngeal cleft repair: The anesthetic perspective. Paediatr Anaesth 23: 334-341.

10. Czinn SJ, Blanchard S (2013) Gastroesophageal reflux disease in neonates and infants: When and how to treat. Paediatr Drugs 15: 19-27.

11. Silva CE, Niedermeier BT, Portinho F (2015) Reflux laryngitis: Correlation between the symptoms findings and indirect laryngoscopy. Int Arch Otorhinolaryngol 19: 234237.

12. Leboulanger N, Garabédian EN (2011) Laryngo-tracheooesophageal clefts. Orphanet J Rare Dis 6: 81. 